PROCEEDINGS OF THE

AMERICAN MATHEMATICAL SOCIETY

Volume 127, Number 5, Pages 1387-1395

S 0002-9939(99)05036-4

Article electronically published on January 28, 1999

\title{
NONOSCILLATION THEOREMS FOR SECOND ORDER NONLINEAR DIFFERENTIAL EQUATIONS
}

\author{
JAMES S. W. WONG
}

(Communicated by Hal L. Smith)

\begin{abstract}
We prove nonoscillation theorems for the second order EmdenFowler equation (E): $y^{\prime \prime}+a(x)|y|^{\gamma-1} y=0, \gamma>0$, where $a(x) \in C(0, \infty)$ and $\gamma \neq 1$. It is shown that when $x^{(\gamma+3) / 2+\delta} a(x)$ is nondecreasing for any $\delta>0$ and is bounded above, then (E) is nonoscillatory. This improves a wellknown result of Belohorec in the sublinear case, i.e. when $0<\gamma<1$ and $0<\delta<(1-\gamma) / 2$.
\end{abstract}

1.

We consider the second order Emden-Fowler equation

$$
y^{\prime \prime}(x)+a(x)|y(x)|^{\gamma-1} y(x)=0, \quad \gamma>0,
$$

on $(0, \infty)$, where $a(x)$ is a positive continuous function. Equation (1) is called superlinear if $\gamma>1$ and sublinear if $0<\gamma<1$. It is known that the superlinear equation has unique solution for any set of given initial conditions and that every solution of the sublinear equation is continuable to the right on $(0, \infty)$. Under the additional assumption that $a(x)$ is locally of bounded variation on $(0, \infty)$, then both the superlinear and the sublinear equations have uniqueness and continuability of solutions. See, e.g., Heidel [9] and Coffman and Wong [4]. To simplify our discussion, we shall assume in this paper that $a(x)$ satisfies this additional assumption so that solutions to equation (1) are unique for given initial conditions and also continuable to the right on $(0, \infty)$.

A solution $y(x)$ of (1) is said to be oscillatory if it has arbitrarily large zeros on $(0, \infty)$, i.e., for any $x_{0} \in(0, \infty)$, there exists $x_{1}>x_{0}$ such that $y\left(x_{1}\right)=0$. Equation (1) is called oscillatory if every solution $y(x)$ has arbitrarily large zeros. On the other hand, equation (1) is called nonoscillatory if every solution has only a finite number of zeros. In view of the nonlinear character of equation (1) when $\gamma \neq 1$ and hence the lack of Sturm's Separation Theorem, this equation may possess both oscillatory and nonoscillatory solutions simultaneously. Here we are concerned with sufficient conditions on the coefficient $a(x)$ so that equation (1) is nonoscillatory. The opposite of nonoscillation of equation (1) is the existence of at least one oscillatory solution. The subject of oscillation for the nonlinear equation has received much attention in the last 40 years; see, e.g., [13] and [17].

Received by the editors August 7, 1997.

1991 Mathematics Subject Classification. Primary 34C10, 34C15.

Key words and phrases. Second order, nonlinear, ordinary differential equations, oscillation, asymptotic behavior. 
To prove the existence of oscillatory solutions of equation (1), it involves monotonicity conditions of the function $\varphi(x)=x^{(\gamma+3) / 2} a(x)$. The well known results are:

Theorem I. If $\varphi(x)$ is nondecreasing in $x$, then equation (1) with $\gamma \neq 1$ has oscillatory solutions.

Theorem II. If $\varphi(x)$ is nonincreasing in $x$ and is also bounded below, then equation (1) with $\gamma \neq 1$ has oscillatory solutions.

Theorem I in the superlinear case, i.e. $\gamma>1$, was first proved by Jasny [11]; a simple proof was later given by Kurzweil [14]. Theorem II in the superlinear case was proved by Erbe and Muldowney [6] as a corollary of a more general theorem which also includes Theorem I for $\gamma>1$. Both Theorems I and II in the sublinear case were proved by Chiou [3].

For nonoscillation results concerning equation (1), sufficient conditions involve the monotonicity of a similar function $\psi(x)=\varphi(x) x^{\delta}=x^{[(\gamma+3) / 2+\delta]} a(x)$ where $\delta>0$. However, in this case results are not as complete as Theorems I and II. In fact, the following two results on superlinear and sublinear equations which were proved 30 years ago still remain to be improved upon:

Theorem III (Kiguradze $1967[12])$. Let $\gamma>1$. If $\psi(x)=x^{[(\gamma+3) / 2+\delta]} a(x)$ where $\delta>0$ is nonincreasing in $x$, then equation (1) is nonoscillatory.

Theorem IV (Belohorec 1967 [2]). Let $0<\gamma<1$. If $\psi(x)=x^{[(\gamma+3) / 2+\delta]} a(x)$ where $0<\delta<(1-\gamma) / 2$ is nondecreasing in $x$ and is also bounded above, then equation (1) is nonoscillatory.

To bring the above theorems in line with the same degree of generality as Theorems I and II, it would be necessary to remove all restrictions on $\delta$ and $\gamma$. Indeed, Kiguradze in his well known survey article [13] proposed his first and major open problem is to extend Theorem III to the sublinear case. Likewise, Erbe and Muldowney called for further improvement on Theorem IV in the sublinear case in the closing remarks of [7].

The purpose of this paper is to give new results on nonoscillation. Our main result is the complete removal of restrictions of $\delta$ and $\gamma$ in Theorem IV, thereby bringing it to the same degree of generality as Theorems I and II. We have not succeeded in obtaining a similar result for Theorem III, so Kiguradze's problem remains open in the sublinear case. However, we are able to establish its validity when $\delta \geq(1-\gamma) / 2$. Furthermore, when $0<\delta<(1-\gamma) / 2$, we show that oscillatory solutions, if they exist, must exhibit certain asymptotic behaviour which lends credence to extending the validity of Theorem III to the sublinear case $0<\gamma<1$. For results related to the nonoscillation theorems discussed in this paper, we also refer the reader to $[1],[4],[5],[6],[8],[15]$ and $[18]$.

2 .

We shall prove nonoscillation theorems concerning equation (1) by studying the transformed equation:

$$
\ddot{w}+(2 \mu-1) \dot{w}+f(t)|w|^{\gamma-1} w+\mu(\mu-1) w=0,
$$


where 'dot' denotes differentiation with respect to $t, \mu$ is a real number $\mu=\frac{1}{2}-$ $\delta /(1-\gamma)$ and

$$
\left\{\begin{array}{l}
w(t)=\frac{y(x)}{x^{\mu}}, \quad t=\log x, \\
f(t)=a(x) x^{2+\mu(\gamma-1)}=a(x) x^{\frac{\gamma+3}{2}+\delta} .
\end{array}\right.
$$

Note that $\mu<\frac{1}{2}$ if $0<\gamma<1$ and $\mu>\frac{1}{2}$ if $\gamma>1$. Since the transformation (3) is "oscillation" invariant, to show that equation (1) is nonoscillatory it is sufficient to prove that the existence of an oscillatory solution $w(t)$ of (2) would lead to a contradiction. Let $w(t)$ be an oscillatory solution of (2), and let $\left\{t_{n}\right\}$ and $\left\{\bar{t}_{n}\right\}$ be sequences of consecutive zeros of $w(t)$ and $\dot{w}(t)$ respectively. Introduce the Pohozaev energy function [16], a technique also used by Heidel and Hinton [10] to study oscillation, as follows:

$$
H(w(t))=\frac{\dot{w}^{2}}{2}+f(t) \frac{|w|^{\gamma+1}}{\gamma+1}+\mu(\mu-1) \frac{w^{2}}{2} .
$$

If $w(t)$ is a solution of (2), then we have

$$
\frac{d}{d t} H(w(t))=(1-2 \mu) \dot{w}^{2}+\dot{f}(t) \frac{|w|^{\gamma+1}}{\gamma+1} .
$$

We are now ready to improve Theorem IV for the sublinear case:

Theorem 1. Let $0<\gamma<1$. If $\psi(x)=x^{(\gamma+3) / 2+\delta} a(x)$ where $\delta>0$ is nondecreasing in $x$ and is also bounded above, then equation (1) is nonoscillatory.

Proof. Let $w(t)$ be an oscillatory solution of (2), and let $\left\{t_{n}\right\}$ and $\left\{\bar{t}_{n}\right\}$ be sequences of zeros of $w(t)$ and $\dot{w}(t)$. Note that $f(t)=\psi(x)$ and $\mu<\frac{1}{2}$, so $H(w(t))$ is nondecreasing by (5). Denote $\lim _{t \rightarrow \infty} H(w(t))=L$ where $L$ could be $+\infty$. Since $H\left(w\left(t_{n}\right)\right)=\frac{1}{2} \dot{w}^{2}\left(t_{n}\right), L$ must be nonnegative. We now show that $0<\gamma<1$ implies $L$ is finite.

Return to equation (1) and set $w(t)=x^{-\mu} y(x)$ to obtain $\dot{w}(t)=x^{1-\mu} y^{\prime}(x)-$ $\mu x^{-\mu} y(x)$. Denote by $\left\{x_{n}\right\}$ the sequence of zeros of $y(x)$ which corresponds to $\left\{t_{n}\right\}$ for $w(t)$. Integrating (1) from $x_{n}$ to $\bar{x}_{n}$ where $y^{\prime}\left(\bar{x}_{n}\right)=0$, we find

$$
y^{\prime}\left(x_{n}\right)=\int_{x_{n}}^{\bar{x}_{n}} a(x) y(x)^{\gamma} d x
$$

Without loss of generality, we may assume $y(x)>0$ on $\left(x_{n}, \bar{x}_{n}\right)$ and $y^{\prime}\left(x_{n}\right)>0$. Since $a(x)>0$, we have $y^{\prime \prime}(x)<0$ and $y(x) \leq y^{\prime}\left(x_{n}\right)\left(x-x_{n}\right)$ on $\left(x_{n}, \bar{x}_{n}\right)$. Using this estimate in $(6)$, we obtain

$$
y^{\prime}\left(x_{n}\right)^{1-\gamma} \leq \int_{x_{n}}^{\bar{x}_{n}} a(x) x^{\gamma} d x \leq K \int_{x_{n}}^{\infty} x^{\frac{\gamma-3}{2}-\delta} d x=K_{1} x_{n}^{\frac{\gamma-1}{2}-\delta}
$$

where $K=\lim _{x \rightarrow \infty} \psi(x)=\lim _{t \rightarrow \infty} f(t)$ and $K=K_{1}[\delta+(1-\gamma) / 2]$. Multiplying (7) by $x_{n}^{(1-\mu)(1-\gamma)}$, we find

$$
\dot{w}\left(t_{n}\right)^{1-\gamma}=x_{n}^{(1-\mu)(1-\gamma)} y^{1-\gamma}\left(x_{n}\right) \leq K_{1} x_{n}^{\frac{1-\gamma}{2}+\delta} x_{n}^{\frac{\gamma-1}{2}-\delta}=K_{1} .
$$

This together with $H\left(w\left(t_{n}\right)\right)=\frac{1}{2} \dot{w}^{2}\left(t_{n}\right)$ shows that $\lim _{t \rightarrow \infty} H(w(t))=L$ is finite.

Having established that $L$ is finite, we integrate (5) from $t_{0}$ to an arbitrary $t$ and find

$$
H(w(t))-H\left(w\left(t_{0}\right)\right)=(1-2 \mu) \int_{t_{0}}^{t} \dot{w}^{2}+\int_{t_{0}}^{t} \dot{f} \frac{|w|^{\gamma+1}}{\gamma+1} .
$$


Since $L$ is finite, $\mu<\frac{1}{2}$ and $\dot{f}(t) \geq 0$, equation (9) shows that $\int^{\infty} \dot{w}^{2}<\infty$. For $\delta \geq(1-\gamma) / 2$ we have $\mu(\mu-1) \geq 0$, so $f(t) \geq f\left(t_{0}\right) \geq 0$ together with $L$ finite imply from (4) that $\dot{w}$ and $w$ are bounded which in turn yields by (2) the boundedness of $\ddot{w}$. Also, $\dot{w} \in L^{2}(0, \infty)$ and $|\ddot{w}| \leq B$ implies $\lim _{t \rightarrow \infty} \dot{w}(t)=0$. However, $\frac{d}{d t} H(w) \geq 0$ implies that $H\left(w\left(t_{n}\right)\right)=\frac{1}{2} \dot{w}\left(t_{n}\right)^{2}$ is nondecreasing which is the desired contradiction.

For $0<\delta<(1-\gamma) / 2$, this is Belohorec's Theorem IV. However, for the sake of completeness, we shall follow the same argument above, hence providing an alternative proof of his result. Let $m_{0}=\mu(1-\mu)>0$. Returning to the transformed equation (2), we observe that the related maxima $\left|\dot{w}\left(\bar{t}_{n}\right)\right|$ satisfies

$$
\ddot{w}\left(\bar{t}_{n}\right)+w\left(\bar{t}_{n}\right)\left[f\left(\bar{t}_{n}\right)\left|w\left(\bar{t}_{n}\right)\right|^{\gamma-1}-m_{0}\right]=0 .
$$

Now maxima $\ddot{w}\left(\bar{t}_{n}\right) \leq 0$ and (10) give that

$$
\left|w\left(\bar{t}_{n}\right)\right| \leq\left[\frac{f\left(\bar{t}_{n}\right)}{m_{0}}\right]^{\frac{1}{1-\gamma}} \leq\left(\frac{K}{m_{0}}\right)^{\frac{1}{1-\gamma}} .
$$

Using (11) and the fact that $\lim _{t \rightarrow \infty} H(w(t))$ exists, we again conclude that $\dot{w}$ and $\ddot{w}$ are bounded. Hence by repeating the same argument as in the last five lines of the preceding paragraph, we obtain the desired contradiction. The proof is now complete.

3.

In this section we prove the analogue of Theorem 1 for the superlinear case, namely,

Theorem 2. Let $\gamma>1$. If $x^{(\gamma+3) / 2+\delta} a(x)$ where $\delta>0$ is nondecreasing in $x$ and is also bounded above, the equation (1) is nonoscillatory.

Proof. We proceed in the same manner as in the proof of Theorem 1 . Here $\gamma>1$, so $\mu>\frac{1}{2}$ and $1-2 \mu<0$. Note that $\mu(\mu-1) \geq 0$ if $\delta \geq(\gamma-1) / 2$ and $\mu(\mu-1)<0$ whenever $0<\delta<(\gamma-1) / 2$. We shall aim to prove the fact that for any oscillatory solution $w(t), \lim _{t \rightarrow \infty} H(w(t))=L$ exists firstly as a finite nonnegative number and in fact must be zero.

We first begin by proving that $H(w(t))$ is bounded from below. When $\mu(\mu-1) \geq 0$, we note that all three terms on the right hand side of equation (4) are nonnegative, hence $H(w(t))$ is nonnegative and is bounded from below. Denote $m_{0}=\mu(1-\mu)>0$, and write $f_{0}=f\left(\bar{t}_{0}\right)>0$. From its definition (4), we note that

$$
H(w(t)) \geq f_{0} \frac{w^{\gamma+1}}{\gamma+1}-\frac{m_{0}}{2} w^{2}=\Phi(w(t)) .
$$

Since $\gamma>1, \Phi(w)$ attains its minimum when $w(t)=\left(m_{0} / f_{0}\right)^{1 /(\gamma-1)}$, namely

$$
\inf _{t \geq 0} \Phi(w(t))=\Phi\left(\left[\frac{m_{0}}{f_{0}}\right]^{\frac{1}{\gamma-1}}\right)=\left[\frac{1-\gamma}{2(\gamma+1)}\right]\left[\frac{m_{0}}{f_{0}}\right]^{\frac{1+\gamma}{\gamma-1}},
$$

which is negative but a finite number. This proves that $H(w(t))$ is bounded below for all $t \geq t_{0}$.

Next to show that $H(w(t))$ is also bounded above, we again consider the two separate cases $\mu(\mu-1) \geq 0$ and $\mu(\mu-1)<0$. 
In the first instance, we have from (4) and (5)

$$
\frac{d}{d t} H(w(t))=(1-2 \mu) \dot{w}^{2}+\dot{f} \frac{w^{\gamma+1}}{\gamma+1} \leq \frac{\dot{f}}{f} H(w(t)) .
$$

Since $\lim _{t \rightarrow \infty} f(t)=K<\infty$, (13) yields boundedness from above of $H(w(t))$. Let $m_{0}=\mu(1-\mu)>0$. We wish to estimate $\Phi(w)$ from below and note that

$$
\Phi(w) \geq f_{0} \frac{w^{\gamma+1}}{\gamma+1}-\frac{m_{0}}{2} w^{2} \geq f_{1} w^{\gamma+1}-\frac{\gamma+1}{2} f_{1} w^{\gamma+1}-m_{1},
$$

where $f_{1}=f_{0}(\gamma+1)^{-1}, m_{1}=[(\gamma-1) /(\gamma+1)] f_{1}^{2 /(1-\gamma)}$. Inequality (14) is derived from an application of Young's inequality to the term $\left(m_{0} / 2\right) w^{2}$ with exponent $(\gamma+1) / 2>1$ as follows:

$$
\begin{aligned}
\frac{m_{0}}{2} w^{2} & =\left(\frac{m_{0}}{2} f_{1}^{-\frac{2}{\gamma+1}}\right)\left(f_{1}^{\frac{2}{\gamma+1}} w^{2}\right) \\
& \leq \frac{2 f_{1}}{\gamma+1} w^{\gamma+1}+\frac{\gamma-1}{\gamma+1} f_{1}^{\frac{2}{1-\gamma}}\left(\frac{m_{0}}{2}\right)^{\frac{1+\gamma}{\gamma-1}}
\end{aligned}
$$

From (14), it is easy to choose suitable positive constants $m_{2}$ and $m_{3}$ such that

$$
w^{\gamma+1} \leq m_{1} H(w)+m_{2} .
$$

Using (15) in (13), one can upon integration easily deduce that $H(w(t))$ is bounded above for all $t$. Boundedness of $H(w(t))$ implies by (15) that $|w(t)| \leq B_{0}$, hence by (4) we also have $|\dot{w}(t)| \leq B_{1}$, for all $t \geq t_{0}$ which yields $|\ddot{w}(t)| \leq B_{2}$ by using the transformed equation $(2)$.

Note that $\int^{\infty} \dot{f} w^{\gamma+1} /(\gamma+1)<\infty$ since $\dot{f} \geq 0$ and $\lim _{t \rightarrow \infty} f(t)=K$. Returning to (5), it follows from the boundedness of $H(w(t))$ that $\int^{\infty} \dot{w}^{2}<\infty$. This in turn implies that $\lim _{t \rightarrow \infty} H(w(t))$ exists as a nonnegative finite number $L$. Since $|w| \leq B_{0},|\dot{w}| \leq B_{1}$ and $|\ddot{w}| \leq B_{2}$, we again conclude from $\int^{\infty} \dot{w}^{2}<\infty$ that $\dot{w}(t) \rightarrow 0$ as $t \rightarrow \infty$. Thus, $\lim _{t \rightarrow \infty} H(w(t))=L=0$.

To establish a desired contradiction, one needs to exhibit a sequence $\left\{\xi_{n}\right\}$ at which points $\dot{w}\left(\xi_{n}\right) \nrightarrow 0$ as $\xi_{n} \rightarrow \infty$. To this end, we need to introduce a special case of equation (2), i.e. when $\mu=1 / 2$,

$$
\ddot{u}+g(t) u|u|^{\gamma-1}-\frac{1}{4} u=0,
$$

where $u(t)=y(x) / \sqrt{x}$. Corresponding to (16), we also introduce the energy function

$$
G(u(t))=\frac{\dot{u}^{2}}{2}+g(t) \frac{u^{\gamma+1}}{\gamma+1}-\frac{u^{2}}{8} .
$$

Let $\sigma=\delta /(\gamma-1)>0$. Then $u(t)=x^{\sigma} w(t)$ and $\dot{u}(t)=x^{\sigma}(\dot{w}+\sigma w)$. We can now relate $G(u(t))$ to $H(w(t))$ by the following identity:

$$
x^{-2 \sigma} G(u(t))=H(w(t))+\sigma w \dot{w} .
$$

Recall that $|w| \leq B_{0}, \dot{w}(t) \rightarrow 0$, and $H(w(t)) \rightarrow 0$ as $t \rightarrow \infty$, hence by (18) we have

$$
\lim _{t \rightarrow \infty} x^{-2 \sigma} G(u(t))=0 .
$$


Let $\xi_{n}$ be relative maxima of $u(t)$ on $\left(t_{n}, t_{n-1}\right)$, i.e. $\dot{u}\left(\xi_{n}\right)>0$ and $u\left(\xi_{n}\right)>0$ with $\ddot{u}\left(\xi_{n}\right) \leq 0$. Using equation (16), we have

which becomes

$$
g\left(\xi_{n}\right)\left|u\left(\xi_{n}\right)\right|^{\gamma-1} \geq \frac{1}{4}
$$

$$
f\left(\xi_{n}\right)\left|w\left(\xi_{n}\right)\right|^{\gamma-1} \geq \frac{1}{4}
$$

Since $\lim _{t \rightarrow \infty} f(t)=K,(21)$ implies $\left|w\left(\xi_{n}\right)\right| \geq(1 / 4 K)^{1 /(\gamma-1)}$. Evaluating (18) at $t=\xi_{n}$, we find

$$
e^{-2 \sigma \xi_{n}} G\left(u\left(\xi_{n}\right)\right)=H\left(w\left(\xi_{n}\right)\right)+\sigma w\left(\xi_{n}\right) \dot{w}\left(\xi_{n}\right)=H\left(w\left(\xi_{n}\right)\right)-\sigma^{2} w^{2}\left(\xi_{n}\right) .
$$

Since $H\left(w\left(\xi_{n}\right)\right) \rightarrow 0$ as $n \rightarrow \infty$, putting (20) in (21) yields

$$
\lim _{n \rightarrow \infty} e^{-2 \sigma \xi_{n}} G\left(u\left(\xi_{n}\right)\right) \leq-\sigma^{2}\left(\frac{1}{4 K}\right)^{2 /(\gamma-1)}<0
$$

which contradicts (19). This completes the proof.

4 .

In this section, we shall prove an analogue of Theorem III in the sublinear case for $\delta \geq(1-\gamma) / 2$. Our result is the following:

Theorem 3. Let $0<\gamma<1$. If $\psi(x)=x^{(\gamma+3) / 2+\delta} a(x)$ where $\delta \geq(1-\gamma) / 2$ is nonincreasing in $x$, the equation (1) is nonoscillatory.

Proof. Let $y(x)$ be an oscillatory solution of (1) and $\left\{x_{n}\right\}$ be the sequence of consecutive zeros of $y(x)$. Using (7), we can estimate as follows:

$$
\left(y^{\prime}\left(x_{n}\right)\right)^{1-\gamma} \leq \int_{x_{n}}^{\infty} a(x) x^{\gamma} d x \leq c_{1} \psi\left(x_{n}\right) x_{n}^{(\gamma-1) / 2-\delta},
$$

where $c_{1}=[\delta+(1-\gamma) / 2]^{-1}>0$, since $\psi(x)$ is nonincreasing. Note that $\psi^{\prime}(x) \leq 0$ also implies $a^{\prime}(x) \leq 0$. We now consider the energy function:

$$
E(y(x))=\frac{y^{\prime 2}}{a}+\frac{2 y^{\gamma+1}}{\gamma+1} .
$$

It is easy to see that for solution $y(x)$ of (1) the derivative $\frac{d}{d x} E(y(x))$ is

$$
\frac{d}{d x} E(y(x))=-\frac{a^{\prime}}{a^{2}} y^{\prime 2} \geq 0 .
$$

From (24), it follows that $\left\{y^{\prime}\left(x_{n}\right) / \sqrt{a\left(x_{n}\right)}\right\}$ forms a nondecreasing sequence, and in particular,

$$
y^{\prime}\left(x_{n}\right) \geq c_{0} a^{\frac{1}{2}}\left(x_{n}\right)
$$

where $c_{0}=y^{\prime}\left(x_{0}\right) / \sqrt{a\left(x_{0}\right)}>0$. Substituting (25) into (22), we find

$$
c_{0}^{1-\gamma} a^{\frac{1-\gamma}{2}}\left(x_{n}\right) \leq c_{1} \psi\left(x_{n}\right) x_{n}^{\frac{\gamma-1}{2}-\delta} .
$$

Multiplying $x_{n}^{\left(\frac{\gamma+3}{2}+\delta\right)\left(\frac{1-\gamma}{2}\right)}$ through (26), we obtain

$$
c_{0}^{1-\gamma}\left(\psi\left(x_{n}\right)\right)^{\frac{1-\gamma}{2}} \leq c_{1} \psi\left(x_{n}\right) x_{n}^{\left(\frac{1+\gamma}{2}\right)\left(\frac{1-\gamma}{2}-\delta\right)} .
$$


Since $\psi\left(x_{n}\right)$ is nonincreasing, letting $n \rightarrow \infty$ in (27) gives the desired contradiction when $\delta>(1-\gamma) / 2$.

We now turn to the case when $\delta=(1-\gamma) / 2$. Here $\mu=0$ and $\psi(x)=x^{2} a(x)$. If $\lim _{t \rightarrow \infty} \psi(x)=0$, then (27) yields $c_{0}^{1-\gamma} \leq c_{1} \psi\left(x_{n}\right)^{(1+\gamma) / 2}$, which gives the desired contradiction. The more difficult case is when $\lim _{t \rightarrow \infty} \psi(x)=k>0$. We need to return to the transformed equation (2) and consider the energy function (4) and its derivative (5) with $\mu=0$, namely,

$$
H(w(t))=\frac{\dot{w}^{2}}{2}+f(t) \frac{w^{\gamma+1}}{\gamma+1},
$$

and

$$
\frac{d}{d t} H(w(t))=\dot{w}^{2}+\dot{f}(t) \frac{w^{\gamma+1}}{\gamma+1}
$$

Next we compute the derivative of $f^{-1} H$ as follows:

$$
\frac{d}{d t} f(t)^{-1} H(w(t))=\dot{w}^{2}\left[f(t)^{-1}-\frac{1}{2} \dot{f}(t) f(t)^{-2}\right] \geq 0
$$

which implies $f^{-1} H(w)$ is nondecreasing and $\lim _{t \rightarrow \infty} f^{-1} H(w)$ exists possibly infinite.

Recall that $f(t)=\psi(x), w(t)=y(x)$ and in this case $\dot{w}(t)=x y^{\prime}(x)$. Returning to (22), we would then have

$$
\left[\dot{w}\left(t_{n}\right)\right]^{1-\gamma}=x_{n} y^{\prime}(x)^{1-\gamma} \leq c_{1} x_{n}^{\frac{\gamma_{1}}{2}-\delta}=c_{1},
$$

since $\delta=(1-\gamma) / 2$. As $f(t)$ is bounded away from zero, we have

$$
\frac{\dot{w}\left(t_{n}\right)^{2}}{f\left(t_{n}\right)} \leq \frac{1}{k} \dot{w}^{2}\left(t_{n}\right) \leq \frac{c_{1}^{2 /(1-\gamma)}}{k}<\infty
$$

Hence (29) implies $\lim _{t \rightarrow \infty} f^{-1} H(w)$ exists as a finite number. Now $\lim _{t \rightarrow \infty} f(t)=$ $k$ implies $\lim _{t \rightarrow \infty} H(w)$ exists as a finite positive number $L$.

Boundedness of $H(w(t))$ implies $|w(t)| \leq B_{0}$ which together with $|\dot{w}(t)| \leq$ $B_{1}$ implies by (2) that $|\ddot{w}(t)| \leq B_{2}$. Furthermore, we also have from (29) that $\left|\int^{\infty} \dot{f} w^{\gamma+1} /(\gamma+1)\right|<\infty$ and $\int^{\infty} \dot{w}^{2}<\infty$. This implies that $\lim _{t \rightarrow \infty} \dot{w}(t)=0$ and $\lim _{t \rightarrow \infty} H(w(t))=L=0$.

The fact $f^{-1} H(w)$ is nondecreasing shows that $f^{-1}\left(t_{n}\right) \dot{w}^{2}\left(t_{n}\right)$ is also nondecreasing. Thus $\dot{w}^{2}\left(t_{n}\right) \geq c_{1} f\left(t_{n}\right) \geq c_{1} k$ where $c_{1}=f^{-1}\left(t_{0}\right) w^{2}\left(t_{0}\right)$, which contradicts the fact that $H(w(t)) \rightarrow 0$ as $t \rightarrow \infty$. The proof of Theorem 3 is now complete.

Our last result deals with the other remaining part of Theorem 3, i.e. when $\psi(x)$ is nonincreasing and $0<\delta<(1-\gamma) / 2$. In this case, we are able to prove the following.

Theorem 4. Let $0<\gamma<1$. If $\psi(x)=x^{(\gamma+3) / 2+\delta} a(x)$ where $0<\delta<(1-\gamma) / 2$ is nonincreasing, then any oscillatory solution $y(x)$ satisfies

$$
\lim _{x \rightarrow \infty} y(x) x^{-\mu}=\lim _{x \rightarrow \infty} x^{1-\mu} y^{\prime}(x)=0,
$$

where $\mu=\frac{1}{2}-[\delta /(1-\gamma)]$. 
Proof. We again work with the transformed equation (2) and let $w(t)$ be an oscillatory solution with $\left\{t_{n}\right\},\left\{\bar{t}_{n}\right\}$ its sequences of consecutive zeros and relative maximas and minimas. Denote $m_{0}=\mu(1-\mu)>0$. Since $\dot{w}\left(\bar{t}_{n}\right)=0$ and $\ddot{w}\left(\bar{t}_{n}\right) \leq 0$, it follows from (2) that

$$
\left|w\left(\bar{t}_{n}\right)\right| \leq\left(\frac{f\left(\bar{t}_{n}\right)}{m_{0}}\right)^{\frac{1}{1-\gamma}}
$$

similarly, when $w\left(\bar{t}_{n}\right)<0$ and $\ddot{w}\left(\bar{t}_{n}\right) \geq 0$, then the above also holds. Hence $w(t)$ is bounded, say $|w| \leq B_{0}$. Furthermore, we note that $H\left(w\left(\bar{t}_{n}\right)\right)$ is bounded from above by

$$
H\left(w\left(\bar{t}_{n}\right)\right)=\frac{f\left(\bar{t}_{n}\right)}{\gamma+1}\left|w\left(\bar{t}_{n}\right)\right|^{\gamma+1}-\frac{m_{0}}{2}\left|w\left(\bar{t}_{n}\right)\right|^{2} \leq\left(\frac{f\left(\bar{t}_{n}\right)}{m_{0}}\right)^{\frac{2}{1-\gamma}}\left\{\frac{1-\gamma}{2(\gamma+1)} m_{0}\right\} .
$$

Note that $|w| \leq B_{0}$ implies $\left|\int^{\infty} \dot{f}(t) w^{\gamma+1} /(\gamma+1)\right|<\infty$. It follows from (31) and (9) that $\lim _{t \rightarrow \infty} H(w(t))=L$ exists where $L$ is a nonnegative finite number. This further implies that $\sup \left|\dot{w}\left(t_{n}\right)\right| \leq B_{0}$, and also that $\int^{\infty} \dot{w}^{2}<\infty$. Once again $|w| \leq B_{0},|\dot{w}| \leq B_{1}$, and by (2) $|\ddot{w}| \leq B_{2}$ imply $\lim _{t \rightarrow \infty} \dot{w}(t)=0$. Note that $H\left(w\left(t_{n}\right)\right)=\frac{1}{2} \dot{w}\left(t_{n}\right) \rightarrow 0$ as $n \rightarrow \infty$, so $L=0$. Since $\dot{w}(t) \rightarrow 0$ as $t \rightarrow \infty$ and $L=0$, we have

$$
\lim _{t \rightarrow \infty} \frac{|w(t)|^{2}}{\gamma+1}\left(f(t)|w(t)|^{\gamma-1}-\frac{(\gamma+1) m_{0}}{2}\right)=0 .
$$

We now consider two separate cases: (a) $\lim _{t \rightarrow \infty} f(t)=0$ and (b) $\lim _{t \rightarrow \infty} f(t)=$ $k>0$. We suppose there exists a sequence $\left\{\eta_{k}\right\}, \eta_{k} \rightarrow \infty$ as $k \rightarrow \infty$, such that $\lim _{k \rightarrow \infty}\left|w\left(\eta_{k}\right)\right|=\alpha>0$. In that case, we evaluate $t$ in (32) at $\eta_{k}$ and find

$$
\lim _{k \rightarrow \infty} \frac{f\left(\eta_{k}\right)\left|w\left(\eta_{k}\right)\right|^{\gamma-1}}{\gamma+1}=\frac{m_{0}}{2}>0
$$

which is incompatible with the assumption that $\lim _{t \rightarrow \infty} f(t)=0$.

Next we consider case (b), and observe from (33) that $\alpha^{\gamma-1}=(\gamma+1) m_{0} / 2 k$. Returning to equation (2), and noting that $\lim _{t \rightarrow \infty} \dot{w}(t)=0$, we find

$$
\begin{aligned}
-\lim _{t \rightarrow \infty} \ddot{w}(t) & =\lim _{t \rightarrow \infty} f\left(\eta_{k}\right) w^{\gamma}\left(\eta_{k}\right)-m_{0} \lim _{k \rightarrow \infty} w\left(\eta_{k}\right) \\
& =k \alpha^{\gamma}-m_{0} \alpha \\
& =\alpha[(\gamma-1) / 2] m_{0}<0,
\end{aligned}
$$

which is incompatible with $\lim _{t \rightarrow \infty} \dot{w}(t)=0$. This shows that $\lim _{t \rightarrow \infty} w(t)=0$ and the proof is completed.

We remark that Belohorec [2] reported that Theorem III remains valid in the sublinear case for all $\delta>0$ provided that $\lim _{x \rightarrow \infty} \psi(x)=k>0$. The proof of Theorem 3 when $\delta=(1-\gamma) / 2$ showed that it is easier to deduce nonoscillation when in fact $\lim _{x \rightarrow \infty} \psi(x)=0$. This is because the decreasing nature of $a(x)$ is faster than when $\psi(x)$ is bounded away from zero as compared to when $\psi(x) \rightarrow 0$ as $x \rightarrow \infty$. Indeed, in such case nonoscillation of equation (1) follows from another result of this author [18]; see also [15]. It should perhaps be pointed out that in case $\delta=0$, i.e. when $\psi(x)=\varphi(x)$ is nonincreasing and bounded away from zero, Chiou [3] had in fact established the existence of oscillatory solutions. Thus, it remains an open problem to prove nonoscillation of equation (1), for $0<\delta<(1-\gamma) / 2$ and $\psi(x)$ nonincreasing, regardless of whether $\psi(x)$ is bounded away from zero or not. 


\section{REFERENCES}

1. S. Belohorec, Oscillatory solutions of certain nonlinear differential equations of second order, Mat. Fyz. Casopis Solven. Akad. Vied. 11 (1961), 250-255. (in Czech).

2. S. Belohorec, On some properties of the equation $y^{\prime \prime}(x)+f(x) y^{\alpha}(x)=0,0<\alpha<1$, ibid. 17 (1967), 10-19. MR 35:5703

3. K. L. Chiou, The existence of oscillatory solutions for the equation $d^{2} y / d t^{2}+q(t) y^{r}=0$, $0<r<1$, Proc. Amer. Math. Soc. 35 (1972), 120-122. MR 46:450

4. C. V. Coffman and J. S. W. Wong, Oscillation and nonoscillation of solutions of generalized Emden-Fowler equations, Trans. Amer. Math. Soc. 167 (1972), 399-434. MR 45:5473

5. C. V. Coffman and J. S. W. Wong, Oscillation and nonoscillation theorems for second order differential equations, Funkcial. Ekvac. 15 (1972), 119-130. MR 48:11662

6. L. H. Erbe and J. S. Muldowney, On the existence of oscillatory solutions to nonlinear differential equations, Annali di Matematica Pure ed Applicata 109 (1976), 23-38. MR 58:1380

7. L. H. Erbe and J. S. Muldowney, Nonoscillation results for second order nonlinear differential equation, Rocky Mountain Math. J. 12 (1982), 635-642. MR 84a:34031

8. H. E. Gollwitzer, Nonoscillation theorems for a nonlinear differential equation, Proc. Amer. Math. Soc. 26 (1970), 78-84. MR 41:3885

9. J. W. Heidel, Uniqueness, continuous and nonoscillation for a second order nonlinear differential equation, Pacific J. Math. 32 (1970), 715-721. MR 41:3886

10. J. W. Heidel and D. B. Hinton, The existence of oscillatory solutions for a nonlinear differential equation, SIAM J. Math. Anal. 3 (1972), 344-351. MR 49:5472

11. M. Jasny, On the existence of an oscillatory solution of the nonlinear differential equation of the second order $y^{\prime \prime}+f(x) y^{2 n-1}=0, f(x)>0$, Casopis Pest Mat. 85 (1960), 78-83. MR 26:408

12. I. T. Kiguradze, A note on the oscillation of solution of the equation $u^{\prime \prime}+a(t)|u|^{n} \operatorname{sgn} u=0^{\prime \prime}$, Casopis Pest Mat. 92 (1967), 343-350. (in Russian). MR 36:4064

13. I. T. Kiguradze, On the oscillatory and monotone solutions of ordinary differential equations, Arch. Math. Scripta Fac. Sci. Nat. 14 (1978), 21-44. MR 80b:34031

14. J. Kurzweil, A note on oscillatory solutions of the equations $y^{\prime \prime}+f(x) y^{2 n-1}=0$, Casopis Pest Mat. 85 (1960), 357-358. (Russian). MR 23:A3322

15. M. K. Kwong and J. S. W. Wong, Nonoscillation theorems for a second order sublinear ordinary differential equation, Proc. Amer. Math. Soc. 87 (1983), 467-474. MR 84b:34039

16. S. I. Pohozaev, Eigenfunctions of the equation $\Delta u+\lambda f(u)=0$, Dokl. Akad. Nauk SSSR 165 (1965), 36-39 (in Russian) and Soviet Math. 6 (1965), 1408-1411 (in English). MR 33:411

17. J. S. W. Wong, On the generalized Emden-Fowler equation, SIAM Review 17 (1975), 339-360. MR 51:3610

18. J. S. W. Wong, Remarks on nonoscillation theorems for a second order nonlinear differential equation, Proc. Amer. Math. Soc. 83 (1981), 541-546. MR 82i:34034

Chinney Investments Ltd., Hong Kong

City University of Hong Kong, Hong Kong 